\title{
2-photon imaging of phagocyte-mediated T cell activation in the CNS
}

\author{
Marija Pesic, ${ }^{1}$ Ingo Bartholomäus, ${ }^{1}$ Nikolaos I. Kyratsous, ${ }^{1}$ Vigo Heissmeyer,2 \\ Hartmut Wekerle,,$^{1,3}$ and Naoto Kawakami1,4
}

\begin{abstract}
${ }^{1}$ Max Planck Institute of Neurobiology, Department of Neuroimmunology, Martinsried, Germany. ${ }^{2}$ Helmholtz Zentrum München, Institute of Molecular Immunology, Munich, Germany. ${ }^{3}$ Hertie Senior Professor and ${ }^{4}$ Institute of Clinical Neuroimmunology, Ludwig-Maximilians Universität, Munich, Germany.
\end{abstract}

\begin{abstract}
Autoreactive $\mathrm{T}$ cells can infiltrate the CNS to cause disorders such as multiple sclerosis. In order to visualize $T$ cell activation in the CNS, we introduced a truncated fluorescent derivative of nuclear factor of activated $T$ cells (NFAT) as a real-time $T$ cell activation indicator. In experimental autoimmune encephalomyelitis, a rat model of multiple sclerosis, we tracked $T$ cells interacting with structures of the vascular blood-brain barrier (BBB). 2-photon imaging documented the cytoplasmic-nuclear translocation of fluorescent NFAT, indicative of calcium-dependent activation of the $T$ cells in the perivascular space, but not within the vascular lumen. The activation was related to contacts with the local antigen-presenting phagocytes and was noted only in $T$ cells with a high pathogenic potential. $T$ cell activation implied the presentation of an autoantigen, as the weakly pathogenic $T$ cells, which remained silent in the untreated hosts, were activated upon instillation of exogenous autoantigen. Activation did not cogently signal long-lasting arrest, as individual $\mathrm{T}$ cells were able to sequentially contact fresh APCs. We propose that the presentation of local autoantigen by BBB-associated APCs provides stimuli that guide autoimmune $T$ cells to the CNS destination, enabling them to attack the target tissue.
\end{abstract}

\section{Introduction}

Brain-specific autoimmune $T$ cells homing to the CNS face a formidable challenge, the blood-brain barrier (BBB), which is a complicated composite of a central endothelial tube, concentrically arranged pericytes and phagocytes, and 2 basal laminas (1). This barrier blocks most of the circulating blood components, but its impermeability is not absolute. Most pertinently, the T cells mediating EAE have developed an elaborate set of sequential interactions with different $\mathrm{BBB}$ components to access the brain tissue.

Most encephalitogenic T cells arrive at the CNS within the leptomeninges, where they attach to the luminal surface of the local small vessels, roll along a short distance, and then crawl before passing through the endothelial wall (2). At this stage, recognition of the autoantigen does not seem to have a major role; however, after leaving the blood vessel, the $T$ cells make serial contacts with perivascular phagocytes and ultimately become activated. Although these phagocytes are distinct from classical DCs, they function as efficient APCs. In particular, as previously shown ex vivo, these phagocytes can present myelin autoantigens acquired from the surrounding CNS tissue $(2,3)$.

These observations led us to speculate that the presentation of autoantigens by perivascular and interstitial phagocytes provides immigrant $T$ cells with the critical cues that direct them into the CNS parenchyma. However, due to technical limitations, direct evidence connecting interactions of T cells with local APCs and following $\mathrm{T}$ cell activation has been lacking to date.

In the present study, we applied a new fluorescent marker of cell activation: a truncated nuclear factor of activated $T$ cells (NFAT) fused to GFP ( $\triangle$ NFAT-GFP) that contains the polypeptide sequence controlling nuclear translocation. 2-photon imaging resolution was sufficient to determine subcellular localization of $\triangle$ NFAT-GFP in vivo, and its translocation kinetics were fast

Conflict of interest: The authors have declared that no conflict of interest exists. Citation for this article: J Clin Invest. 2013;123(3):1192-1201. doi:10.1172/JCI67233. enough to investigate functional $\mathrm{T}$ cell interactions with different structures of the target milieu. We used this construct to elucidate the interactions between T cells and APCs within the CNS leptomeninges, the main portal for CNS migrant encephalitogenic T cells. This approach allowed us to demonstrate that perivascular phagocytes, not endothelial cells, activate the incoming $\mathrm{T}$ cells. Furthermore, our results emphasized the effect of autoantigen availability on the locomotor behavior and pathological capacity of CNS autoimmune T cells.

\section{Results}

$\triangle N F A T-G F P-$ expressing T cells. We applied a GFP-labeled, truncated variant of NFAT1 as a "functional" tag to visualize the real-time activation events by which CNS autoimmune T cells cross the leptomeningeal BBB, the essential portal to the CNS parenchyma (3-6). The construct, $\triangle$ NFAT-GFP, contained the regulatory domain of NFAT1 that is necessary for phosphorylation, cytoplasmic sequestration, and calcium-induced and calcineurin-mediated dephosphorylation. Dephosphorylation induces a conformational change, which exposes a nuclear localization signal leading to cytoplasmic-nuclear translocation $(7,8)$. The NFAT construct was truncated to delete the DNA-binding domain of native NFAT (Figure $1 \mathrm{~A}$ and ref. 9), so as not to interfere with gene regulation by endogenous NFAT. Upon $\mathrm{T}$ cell activation, $\triangle$ NFAT-GFP was translocated from the cytosol to the nucleus (Figure 1, B and C), similar to native NFAT1 (10). Cytoplasmic-nuclear translocation of $\triangle$ NFAT-GFP happened within minutes upon ionomycin stimulation; however, reverse transport from nucleus to cytosol after removing activating stimulus took much longer, around 1 hour (Figure 1, C and D, and Supplemental Video 1; supplemental material available online with this article; doi:10.1172/JCI67233DS1). Most importantly, $\triangle$ NFATGFP-expressing T cells behaved like their GFP-expressing counterparts; in particular, their encephalitogenic potential remained unimpaired (Figure 1E). We concluded that $\triangle$ NFAT-GFP serves as a reliable genetic indicator of $\mathrm{T}$ cell activation for 2-photon imaging. 
A

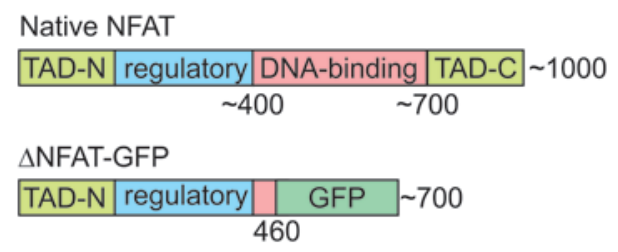

B

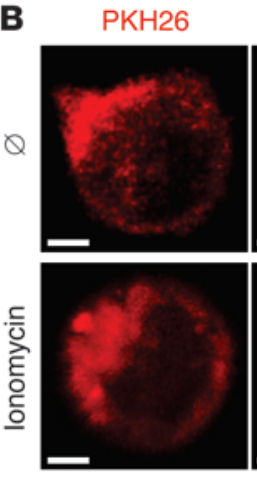

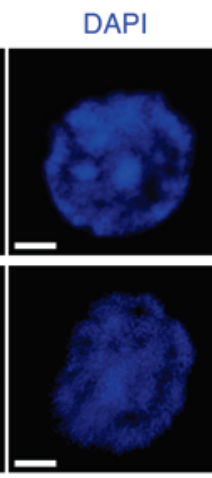
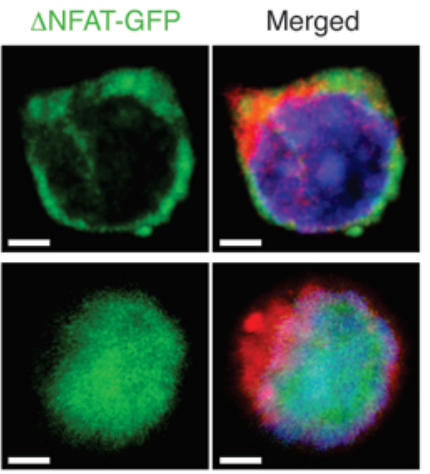

\section{C}
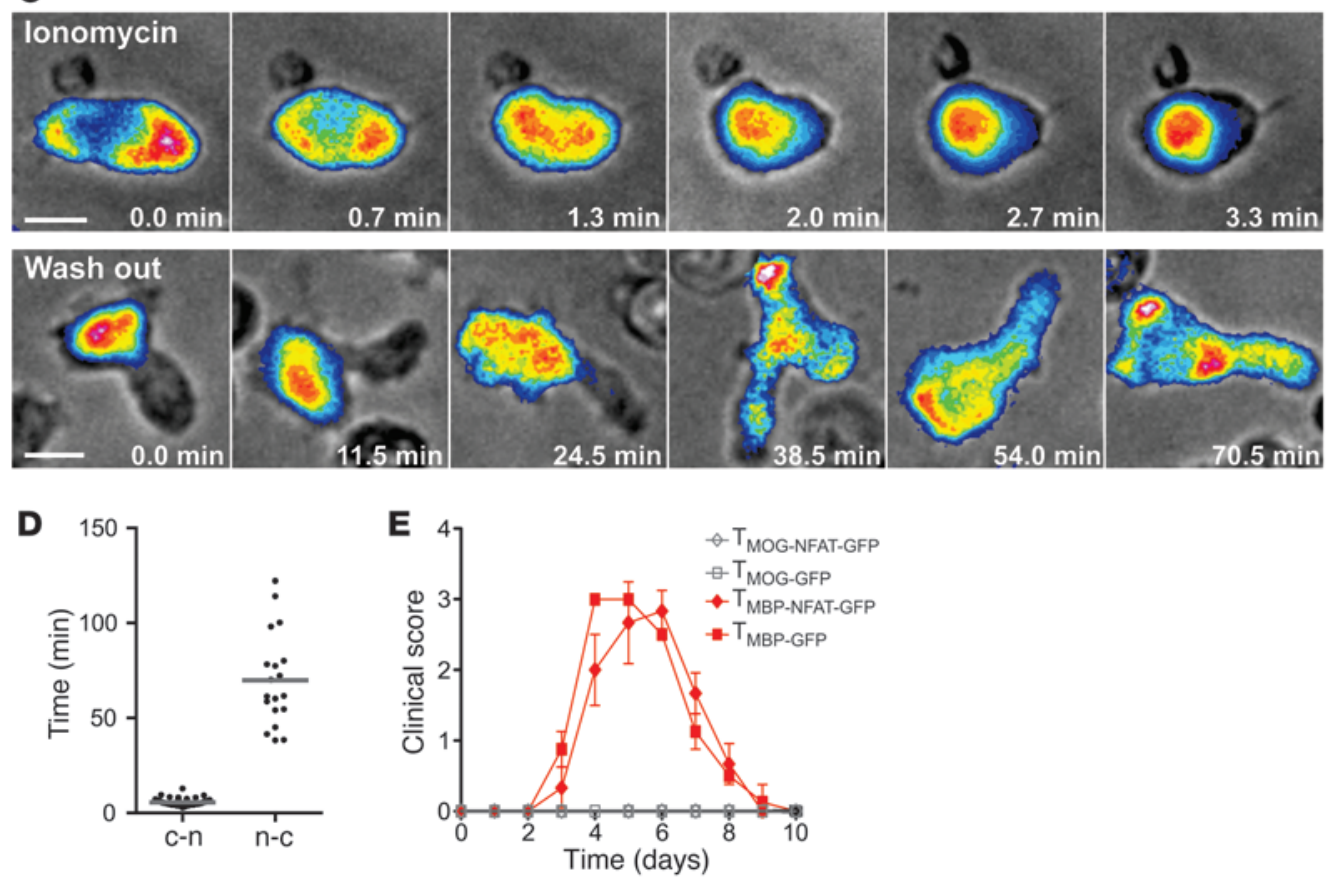

\section{Figure 1}

$\triangle$ NFAT-GFP as an activation marker of T cells. (A) Native and truncated versions of NFAT. aa numbers are indicated. TAD, transcription activation domain. (B) Confocal images of resting in vitro TMBP-NFAT-GFP cells stained with membrane dye PKH26 and DAPI, before and after application of ionomycin. Scale bars: $2 \mu \mathrm{m}$. (C) Time kinetics of cytoplasmic-nuclear (c-n) $\triangle$ NFAT-GFP translocation upon ionomycin stimulation, and reverse nuclear-cytoplasmic ( $n-c)$ transport after ionomycin washout, in representative resting in vitro TMBP-NFAT-GFP cells. Bright field images (gray) were overlaid with pseudocolor images depicting GFP intensity distribution, from blue (low intensity) to red (high intensity). Numbers denote the relative time after addition (top) or washing (bottom) of ionomycin. Scale bars: $5 \mu \mathrm{m}$. (D) Cytoplasmic-nuclear $\Delta$ NFAT-GFP translocation times after addition of ionomycin, and nuclear-cytoplasmic translocation times after ionomycin washout. Each symbol represents a single cell; results are the sum of at least 3 independent experiments per condition. (E) EAE clinical course induced by transfer of $T_{\text {MOG-GFP }}$ or $T_{\text {MBP-GFP }}$ cells as well as $\mathrm{T}_{\text {MOG-NFAT-GFP }}$ or $\mathrm{T}_{\text {MBP-NFAT-GFP }}$ cells. Mean \pm SD from at least 3 animals per group are shown. Representative data from 3 independent experiments per cell line.

The encephalitogenic potential of autoreactive $T$ cells depends on their antigen specificity. Myelin basic protein-specific (MBP-specific) $\mathrm{T}$ cells (referred to herein as $\mathrm{T}_{\mathrm{MBP}}$ cells) are strongly activated in the CNS and trigger classical acute EAE, thus qualifying as EAE ${ }^{\text {hi }}$ $\mathrm{T}$ cells. In contrast, myelin oligodendrocyte glycoprotein-specific (MOG-specific) T cells ( $\mathrm{T}_{\mathrm{MOG}}$ cells) display no activation and hardly mediate any clinical defects; these are EAE ${ }^{\text {lo }} \mathrm{T}$ cells (ref. 5 and Figure 1E). This discrepancy suggested that these 2 cell lines represent good candidates for the study of differential $\mathrm{T}$ cell activation in vivo.

Activation status of intraluminal $T$ cells. The first contact between encephalitogenic T cells and the BBB occurs on the luminal sur- face of the endothelial tube. The T cells attach to the intraluminal surface of leptomeningeal vessels, roll along a short distance, and then continue to crawl on the endothelial cells. Both modes of motion differ in the molecules used for the endothelial contacts. While rolling occurs along the blood stream and involves transient interactions between selectins and glycoproteins (11), crawling is often against the direction of blood flow and depends on the binding of integrins VLA-4 and LFA-1 (2). These interactions appear to involve some $\mathrm{T}$ cell stimulation (12); however, it is not clear which of the endothelial contacts trigger $T$ cell activation, and what extent of stimulation is provided to $\mathrm{T}$ cells within the vascu- 

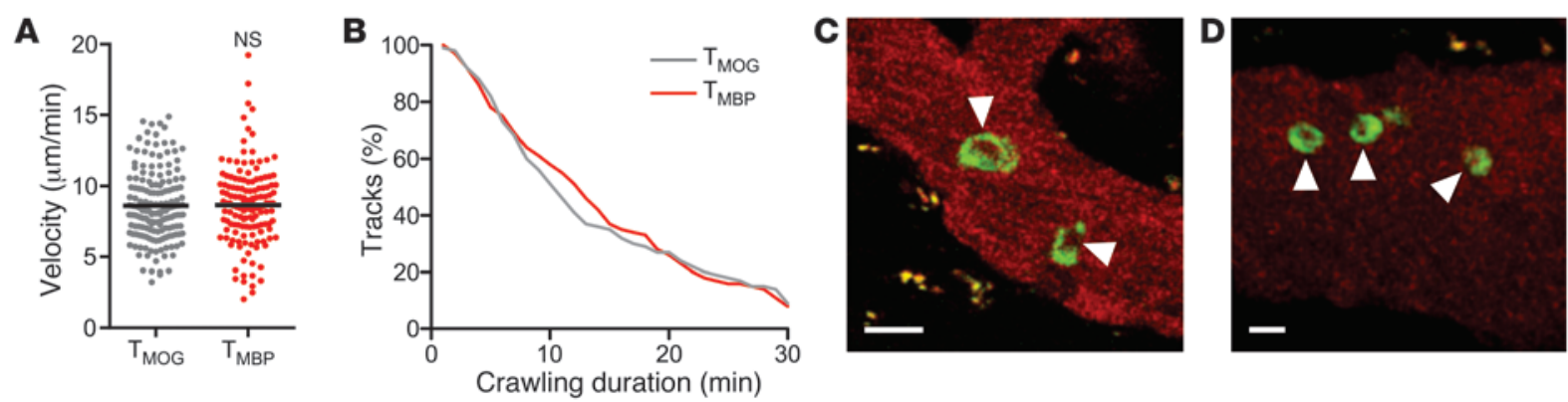

\section{Figure 2}

Activation status of autoreactive T cells within leptomeningeal blood vessels. (A and B) Intraluminal velocity (A) and average crawling time (B) of $T_{\text {MOG-GFP }}$ or $T_{\text {MBP-GFP }}$ cells. Results are sum of 5 different experiments per cell line. (C and $\left.\mathbf{D}\right)$ Representative still images of crawling (C; from

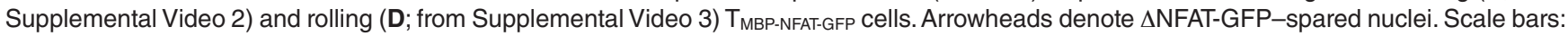
$10 \mu \mathrm{m}$. Significance of differences was determined by Mann-Whitney nonparametric $t$ test.

lar lumen. The results of our 2-photon study were unambiguous: regardless of their pathogenic potential $\left(\mathrm{EAE}^{\mathrm{hi}}\right.$ versus $\left.\mathrm{EAE}^{\mathrm{lo}}\right)$, the $\mathrm{T}$ cells crawled with similar velocities and for similar durations (Figure 2, A and B), and neither the crawling nor the rolling $\mathrm{T}$ cells showed $\triangle$ NFAT-GFP in their nuclei (Figure 2, C and D, and Supplemental Videos 2 and 3 ). We concluded that, if there is any activation of intraluminal $\mathrm{T}$ cells, it is not sufficient to translocate NFAT into the nucleus and, implicitly, to initiate gene activation.

T cell/APC contact after extravasation. Having crossed the BBB, autoimmune T cells promptly contact local APCs. To characterize these interactions, we initially compared the EAE ${ }^{\text {hi }} \mathrm{T}_{\mathrm{MBP}}$ and $\mathrm{EAE}^{\text {lo }} \mathrm{T}_{\mathrm{MOG}}$ cells labeled with a traditional, static GFP (referred to herein as $\mathrm{T}_{\text {MBP-GFP }}$ and $\mathrm{T}_{\text {MOG-GFP }}$ cells, respectively) to outline their locomotion (2). $T_{\text {MBP-GFP }}$ cells moved within the leptomeningeal space with lower velocity and mean square displacement than did $\mathrm{T}_{\text {MOG-GFP }}$ cells (Figure 3, A and B). We next examined T cell/APC contacts for these 2 $\mathrm{T}$ cell lines. These APCs exhibit mostly macrophage and not DC markers, and a substantial proportion expresses MHC class II molecules on their surface, which qualifies them as APCs (2). $T_{\text {MOG-GFP }}$ cells largely ignored the local APCs, moving continuously along straight trajectories (white lines in Figure 3C and Supplemental Video 4). In contrast, $\mathrm{T}_{\mathrm{MBP} \text {-GFP }}$ cells halted upon contact with the APCs, drawing trajectories that twisted around an anchoring point (white lines in Figure 3C and Supplemental Video 5). Contacts of $\mathrm{T}_{\mathrm{MBP}-\mathrm{GFP}}$ cells with APCs were significantly longer-lasting than those of $\mathrm{T}_{\text {MOG-GFP }}$ cells (Figure 3D). Confirming previous reports $(2,5)$, $\mathrm{T}_{\mathrm{MBP}-\mathrm{GFP}}$ cells in leptomeninges and parenchyma upregulated the activation marker OX-40, but $\mathrm{T}_{\text {MOG-GFP }}$ cells did not (Figure $3 \mathrm{E}$ ).

$\triangle N F A T-G F P$ translocation in perivascular $T$ cells. To link the contact-dependent changes in $\mathrm{T}$ cell locomotion with cell activation directly, we compared EAE ${ }^{\text {hi }} \mathrm{T}_{\mathrm{MBP}}$ and EAE ${ }^{\text {lo }} \mathrm{T}_{\mathrm{MOG}}$ cells expressing $\triangle$ NFAT-GFP (referred to herein as $\mathrm{T}_{\text {MBP-NFAT-GFP }}$ and $\mathrm{T}_{\text {MOG-NFAT-GFP }}$ cells, respectively). In order to enhance visualization of the marker's subcellular location, we counterstained the cells with SNARF-1 (13), thus labeling both cytosol and nucleus. To avoid dilution of SNARF-1 staining, we labeled $\mathrm{CD}^{+}{ }^{+} \mathrm{T}$ cells isolated from spleen 3 days after transfer of $\triangle$ NFAT-GFP-expressing $T$ cells, then transferred them into WT recipients (Supplemental Figure 1). Transferred $\mathrm{CD}^{+} \mathrm{T}$ cell-enriched splenocytes penetrated into the CNS meninges more rapidly than did in vitro activated $\mathrm{T}$ cells (14). SNARF-1 counterstaining allowed for determination of $\triangle$ NFATGFP location in up to $90 \%$ of all of cells and facilitated the dis- tinction of $3 \triangle$ NFAT-GFP localization patterns (Figure 4A): cytoplasmic, located in the cytosol and not the nucleus (indicative of resting T cells); nuclear, located in the nucleus and not the cytosol (indicative of activated T cells); and localization in both cytosol and nucleus (indicative of transitional $\mathrm{T}$ cells).

Importantly, in contrast to their intraluminal counterparts, more than $30 \%$ of extravascular $\mathrm{T}_{\text {MBP-NFAT-GFP cells exhibited }}$ nuclear localization of $\triangle$ NFAT-GFP, and another $20 \%$ showed the marker in both cytosol and nucleus. Conversely, $\mathrm{T}_{\text {MOG-NFAT-GFP }}$ cells restricted $\triangle$ NFAT-GFP to the cytosol (Figure 4, B and C, and Supplemental Video 6). Nuclear translocation of $\triangle$ NFAT-GFP was tightly related to the locomotor behavior of the T cells. Regardless of antigen specificity, $T$ cells with cytoplasmic $\triangle$ NFAT-GFP were highly motile, with nearly straight trajectories; in contrast, $T$ cells with nuclear $\triangle$ NFAT-GFP showed intercalated segments of coiled tracks (Figure 4D). In addition, T cell velocity directly correlated with $\triangle$ NFAT-GFP localization. T cells with cytoplasmic $\triangle$ NFAT-GFP moved at an average of $10 \mu \mathrm{m} / \mathrm{min}$, again regardless of antigen specificity, whereas $\mathrm{T}$ cells with nuclear $\triangle$ NFATGFP traveled at a slow velocity (mean, $4.5 \mu \mathrm{m} / \mathrm{min}$ ), and those with both nuclear and cytoplasmic $\triangle$ NFAT-GFP moved with an intermediate speed (mean, $6.5 \mu \mathrm{m} / \mathrm{min}$; Figure 4E). The change in $T$ cell velocity correlated with the change in $\triangle$ NFAT-GFP localization (Figure 4F and Supplemental Video 7).

$T$ cell/APC contacts are crucial for $T$ cell activation. Nuclear translocation of $\triangle$ NFAT-GFP in the T cells correlated with their contacts to local APCs (Figure 5A and Supplemental Video 8). Furthermore, continuous APC contacts (average duration, 20 minutes) were required to transport $\triangle$ NFAT-GFP from the cytosol to the nucleus, whereas brief, transient interactions (average duration, 3 minutes) maintained $\triangle$ NFAT-GFP in the cytosol, regardless of T cell antigen specificity (Figure 5B). It is noteworthy that the cytoplasmic-nuclear translocation of $\triangle$ NFAT-GFP occurred shortly after contact with an APC (average interval, 4 minutes), whereas the average duration of this contact amounted to 20 minutes (Figure $5 \mathrm{C}$ ). In contrast to this downstream translocation, the reverse nuclear-cytoplasmic transport of $\triangle$ NFAT-GFP required a longer period of time: once detached from the APCs, $\triangle$ NFAT-GFP was gradually translocated back to the cytosol, a process requiring approximately 30 minutes (Figure 5, D and E, and Supplemental Video 9). These results correlated to in vitro calculated kinetics of translocation (Figure 1, C and D). 

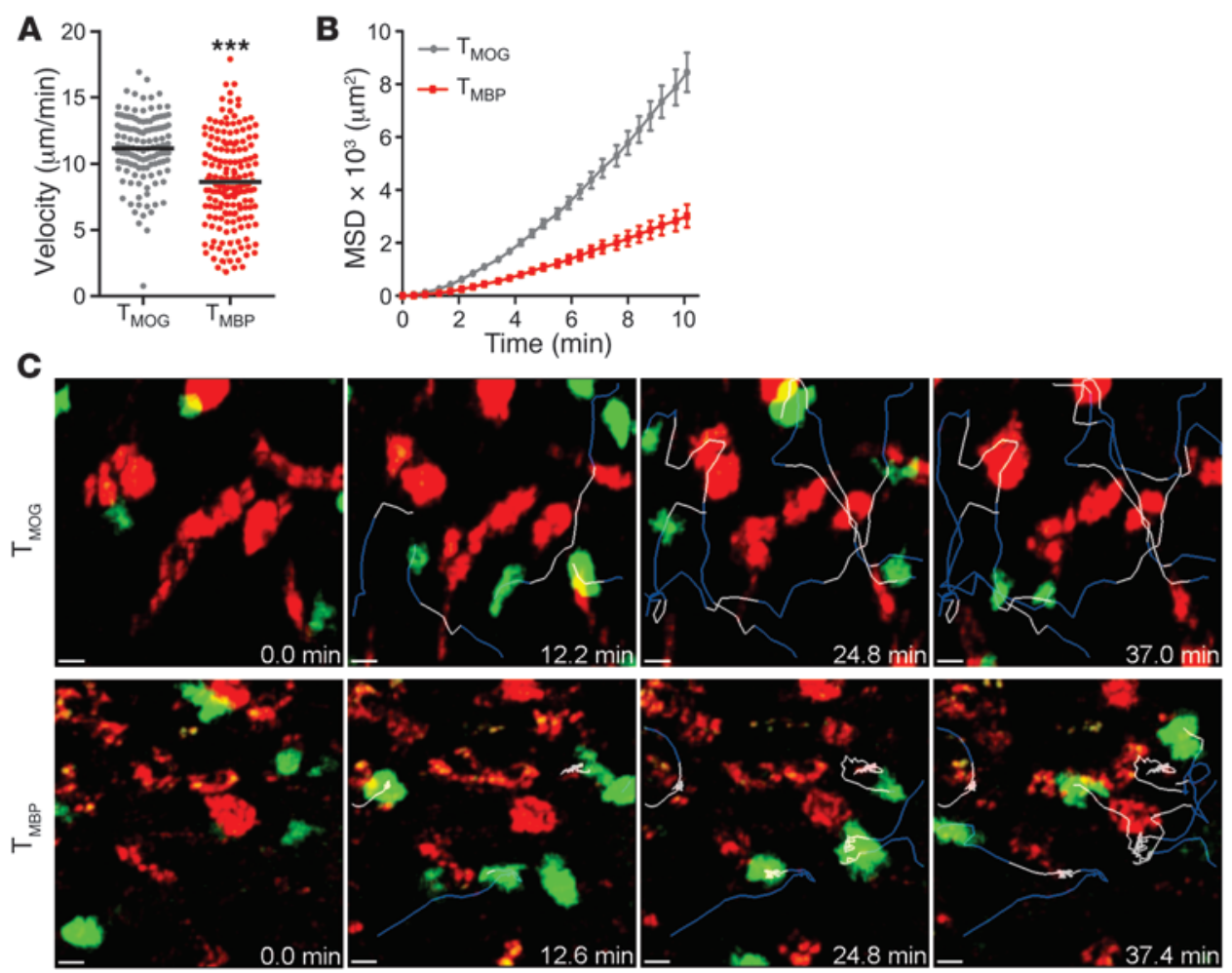

\section{Figure 3}

Motility of EAE hi $T_{M B P-G F P}$ and EAE $E^{\text {lo }}$

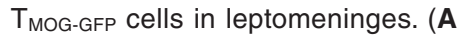
and $\mathbf{B})$ Velocity (A) and mean square displacement (MSD; B) of $\mathrm{T}_{\text {MOG-GFP }}$ or $T_{M B P-G F P}$ cells calculated from intravital recordings. (C) Still images of representative T cell/APC contacts in the leptomeninges: $T_{\text {MOG-GFP }}$ (from Supplemental Video 4) and $T_{\text {MBP-GFP (from Supplemental Video }}$ 5) cells are shown in green, together with local APCs (red). Blue trajectory lines denote no contact; white trajectory lines denote contact. Numbers denote relative time after start of acquisition. Scale bars: $10 \mu \mathrm{m}$. (D) T cell/APC contact duration of $T_{M O G-G F P}$ or $T_{\text {MBP-GFP }}$ cells calculated from intravital recordings. (E) Expression of the activation marker OX-40 on CNS-infiltrating $T$ cells. Results are the sum ( $\mathbf{A}$ and $\mathbf{D})$ or are representative ( $\mathbf{B}$ and $\mathbf{E}$ ) of at least 3 independent experiments per cell line. ${ }^{* * \star} P<0.0001$, Mann-Whitney nonparametric $t$ test.
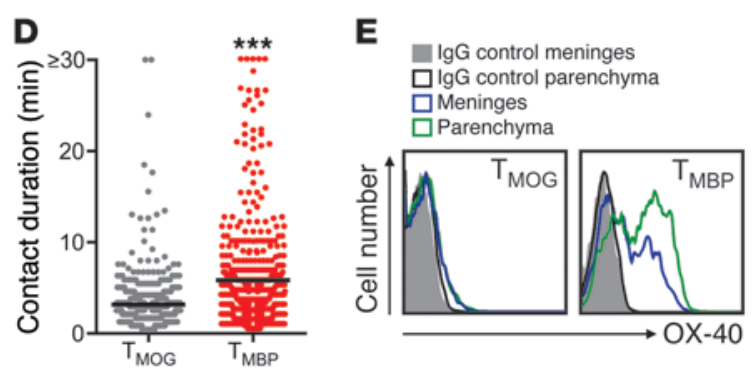

Contacts between T cells and APCs were promiscuous; some APCs could engage several T cells sequentially. The same APC was observed to interact with 2 distinct T cells, in both cases triggering $\triangle$ NFAT-GFP translocation (Figure 5A and Supplemental Video 8). In fact, sequential $T$ cell activation by a single APC was not unusual. Conversely, individual T cells visited several APCs over time. Although most enduring APC contacts were established by the $\mathrm{T}$ cells showing cytoplasmic-nuclear $\triangle$ NFAT-GFP translocation, we also observed extended APC interactions by $\mathrm{T}$ cells with preexisting nuclear $\triangle$ NFAT-GFP (average duration, 10 minutes; Figure $5 \mathrm{~B}$ ). These findings suggest that already-activated $\mathrm{T}$ cells visit successively local APCs and that these visits either maintain their activation state at a particular level or escalate it continuously.

Finally, we examined sequential versus stable T cell/APC interactions for their capacity to induce $T$ cell activation. Nonactivating and activating contacts were distinguished by $\triangle$ NFAT-GFP localization: nonactivating contacts were shorter-lasting (average duration, 3 minutes; Supplemental Figure 2) than the activating ones (average duration, 20 minutes; Figure 5B and Supplemental Figure 2), and during nonactivating contacts, T cells maintained their high velocity (average, $9 \mu \mathrm{m} / \mathrm{min}$; Supplemental Figure 2). Although we cannot exclude that the cells ultimately receive some stimulus after these sequential APC visits, our results indicate that long-lasting contacts are more important for T cell activation.

Antigen availability and $T$ cell activation in the CNS meninges. The discrepant interactions of the $\mathrm{EAE}^{\text {hi }}$ and $\mathrm{EAE}^{\text {lo }} \mathrm{T}$ cell lines with CNS APCs may reflect a difference in the availability of presented autoantigen. It is known that the myelin autoantigen is presented within the local tissue, yet the amounts of antigen are suboptimal, below saturating concentrations. In fact, addition of exogenous autoantigen enhances $\mathrm{T}_{\text {MBP-GFP }}$ and $\mathrm{T}_{\text {MOG-GFP }}$ cell activation and aggravates clinical EAE $(4,5)$. To explore the effect of autoantigen

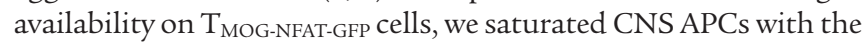
exogenous autoantigen MOG by local microcapillary infusion.

The effect of MOG on $\mathrm{T}_{\text {MOG-NFAT-GFP }}$ cell behavior and $\triangle$ NFATGFP localization was drastic and immediate. Injection of MOG affected the locomotion of $\mathrm{T}_{\text {MOG-NFAT-GFP }}$ cells and also prompted the nuclear translocation of $\triangle$ NFAT-GFP in up to $60 \%$ of cells analyzed (Figure 6, A-C, and Supplemental Video 10), which was more than in $\mathrm{T}_{\text {MBP-NFAT-GFP }}$ cells without the exogenous antigen (Figure 4C). Similar to $T_{\text {MBP-NFAT-GFP }}$ cells, $T_{\text {MOG-NFAT-GFP }}$ cells showing nuclear $\triangle$ NFAT-GFP localization displayed reduced velocity (Figure 6D). In contrast, the motility of $\mathrm{T}_{\text {MOG-NFAT-GFP }}$ cells having cytoplasmic $\triangle$ NFAT-GFP did not differ before and after MOG infusion (Figure 6, 
A
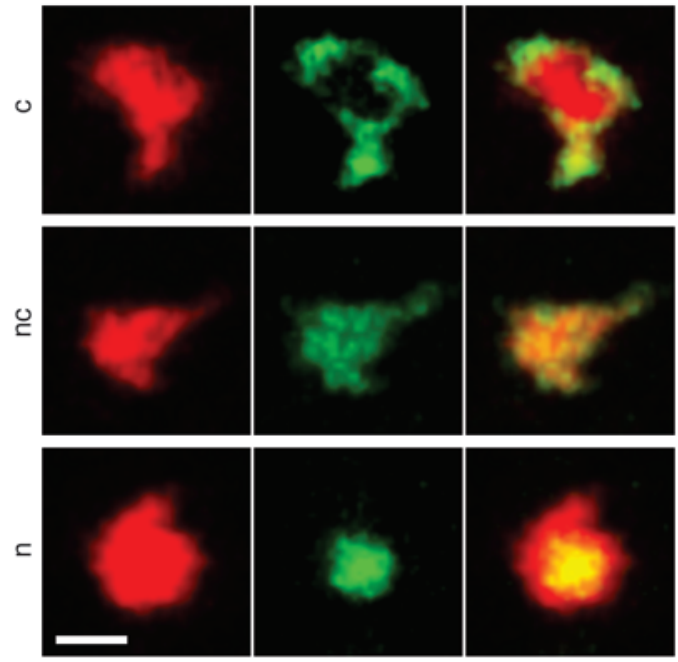

B

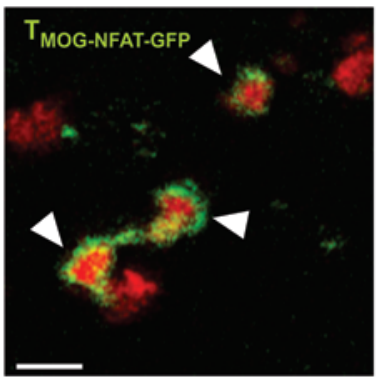

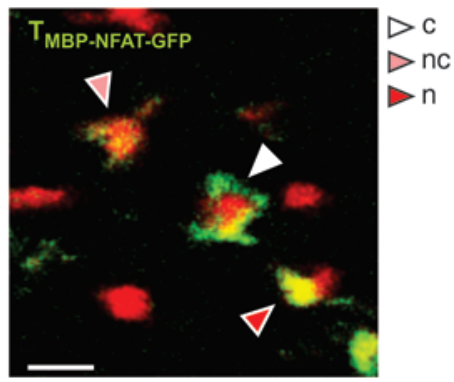
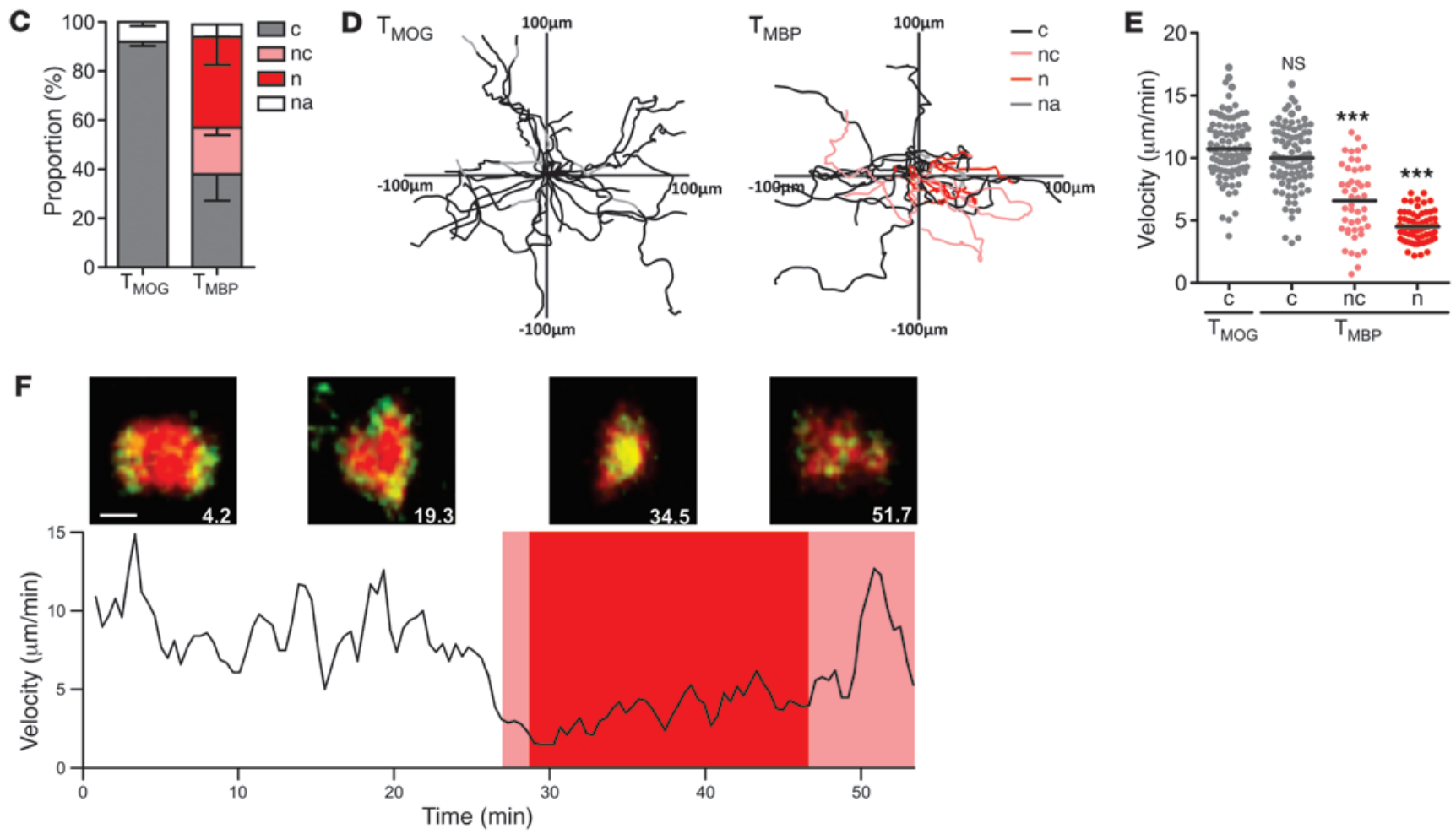

\section{Figure 4}

In vivo T cell activation, shown by nuclear translocation of the $\triangle$ NFAT-GFP marker. (A) 3 patterns of $\triangle$ NFAT-GFP (green) and SNARF-1 (red) in double-labeled T cells: cytoplasmic (c), nuclear ( $n$ ), and nuclear/cytoplasmic ( $\mathrm{nc}$ ). Scale bar: $5 \mu \mathrm{m}$. (B) Still images of TMOG-NFAT-GFP Or TMBP-NFAT-GFP

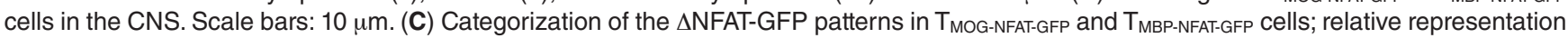
was calculated. na, not analyzable. (D) T cell trajectories from representative movies were superimposed on the coordinates; each line starts at 0. $\triangle$ NFAT-GFP location is denoted by line color. (E) Velocity of T cells with different $\triangle$ NFAT-GFP locations. (F) Velocity (averaged over 3 time points) of representative $T_{\text {MBP-NFAT-GFP }}$ cells changing $\triangle$ NFAT-GFP location. Light and dark red shading denotes $\triangle$ NFAT-GFP in the nucleus/cytosol and in the nucleus, respectively. Images above show $\triangle$ NFAT-GFP localization (green) in a representative SNARF-1-labeled (red) TMBP-NFAT-GFP cell at the indicated times (in minutes; from Supplemental Video 7). Scale bar: $5 \mu \mathrm{m}$. Data in $\mathbf{C}$ and $\mathbf{E}$ are the sum of at least 3 independent experiments and 4 different videos per cell line. ${ }^{* \star *} P<0.001$, 1-way ANOVA followed by Kruskal-Wallis/Dunn multiple-comparison test.

$\mathrm{B}$ and D). The contacts that led to cytoplasmic-nuclear $\triangle \mathrm{NFAT}$ GFP translocation were long-lasting, as were contacts made by $\mathrm{T}$ cells that had already acquired activation (i.e., nuclear localization; Figure 6E). This suggests that the T cells that displayed nuclear $\triangle$ NFAT-GFP were contacting the APCs to maintain their activation status, similar to the APC interactions observed with MBP-specific $\mathrm{T}$ cells. Because NFAT nuclear translocation leads to gene transcription, we confirmed $\mathrm{T}$ cell activation using flow cytometry. As 

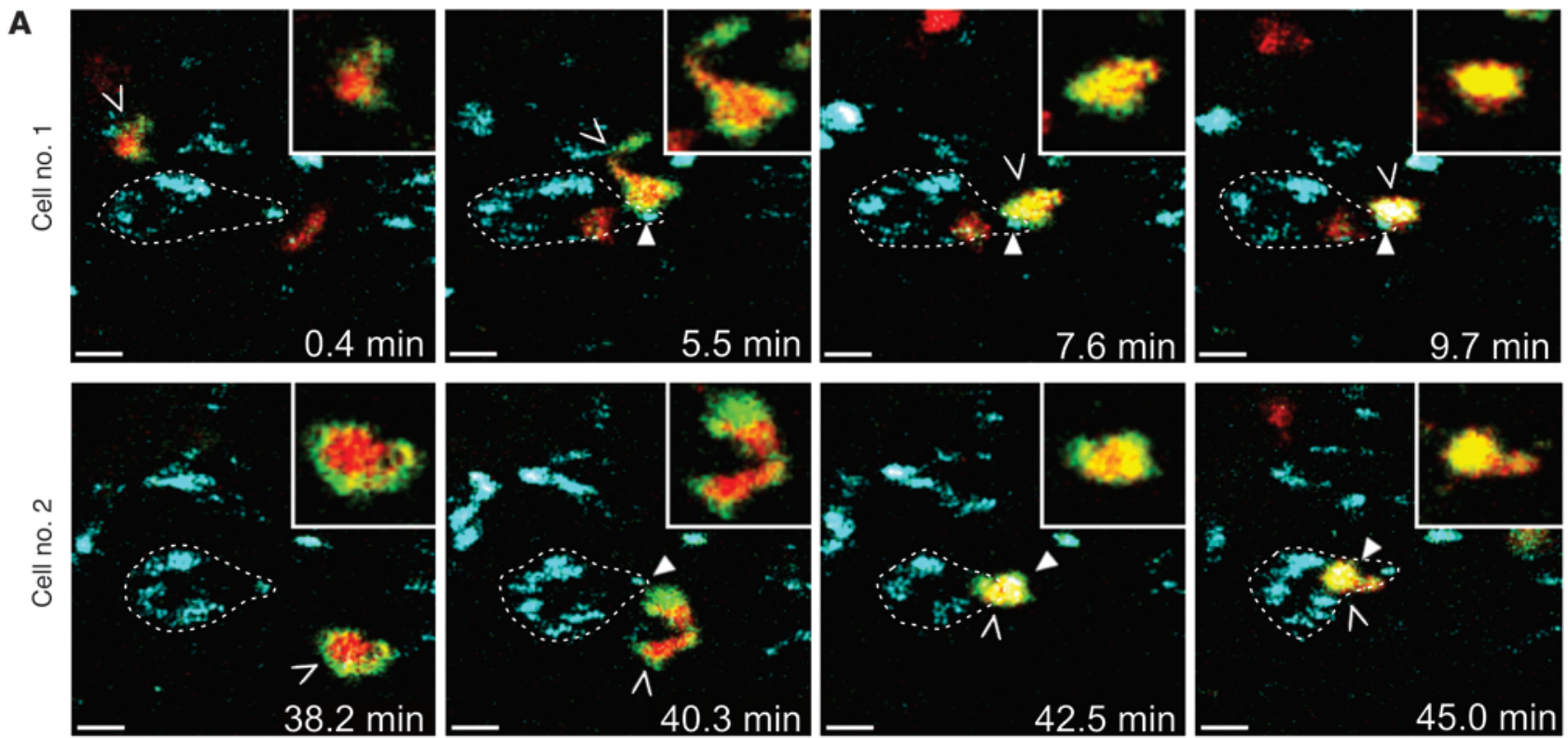

B

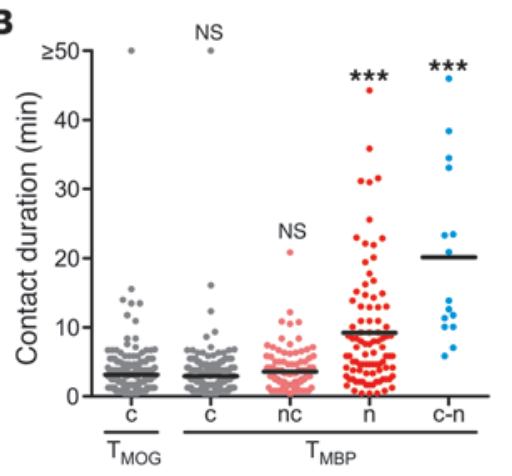

E

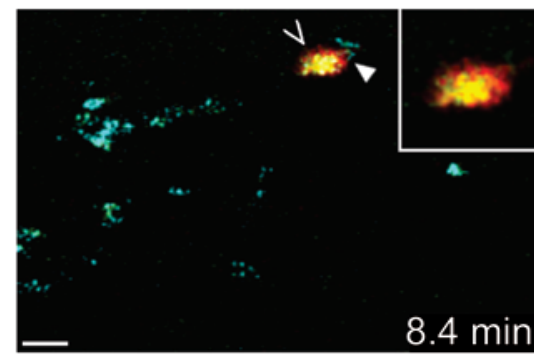

C

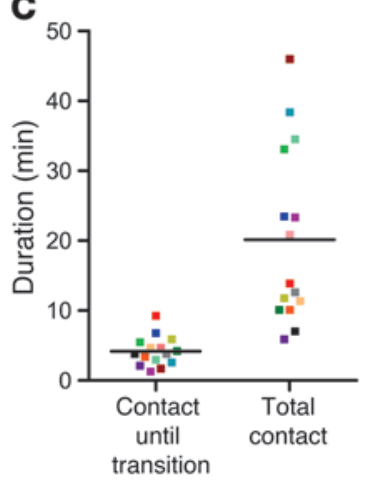

D

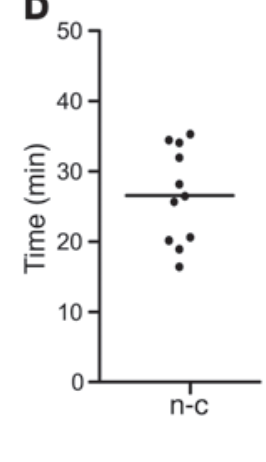

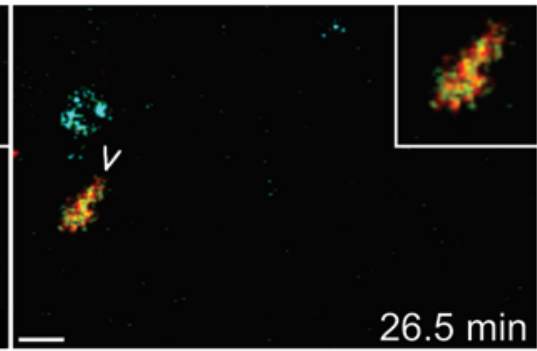

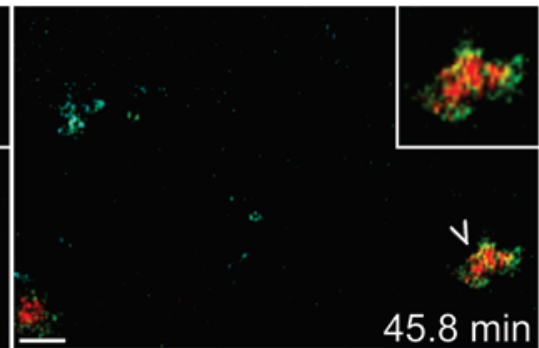

Figure 5

T cell interaction with local APCs. (A) 2 SNARF-labeled (red) $T_{\text {MBP-NFAT-GFP }}$ cells were successively activated (nuclear translocation of $\Delta$ NFAT-GFP; green) after contact with the same APC (cyan). Speculated outlines are shown by dotted lines. From Supplemental Video 8. Relative time after start of acquisition is indicated. Closed arrowheads denote T cell/APC interaction; T cells of interest (open arrowheads) are shown in the insets (green and red channels only). Scale bars: $10 \mu \mathrm{m}$. (B) Duration of T cell/APC contacts by cells with different $\Delta$ NFAT-GFP locations and of contact leading to cytoplasmic-nuclear $\triangle$ NFAT-GFP translocation. (C) Time required for cytoplasmic-nuclear $\triangle$ NFAT-GFP translocation, starting at the beginning of the T cell/APC contact, compared with the total duration of the same contact. Each color represents the same contact. (D) Time required for nuclear-cytoplasmic $\triangle$ NFAT-GFP translocation. Each symbol represents a single cell. (E) SNARF-labeled (red) T $T_{\text {MBP-NFAT-GFP (green) }}$ cell undergoing nuclear-cytoplasmic $\triangle$ NFAT-GFP translocation after detachment from a local APC (cyan). From Supplemental Video 9. Relative time after start of acquisition is indicated. Closed arrowheads denote T cell/APC interaction; T cells of interest (open arrowheads) are shown in the insets (green and red channels only). Scale bars: $10 \mu \mathrm{m}$. Results in B-D are the sum of 3 independent experiments per cell line. ${ }^{* * *} P<0.001$, 1-way ANOVA followed by Kruskal-Wallis/Dunn multiple-comparison test. 
A
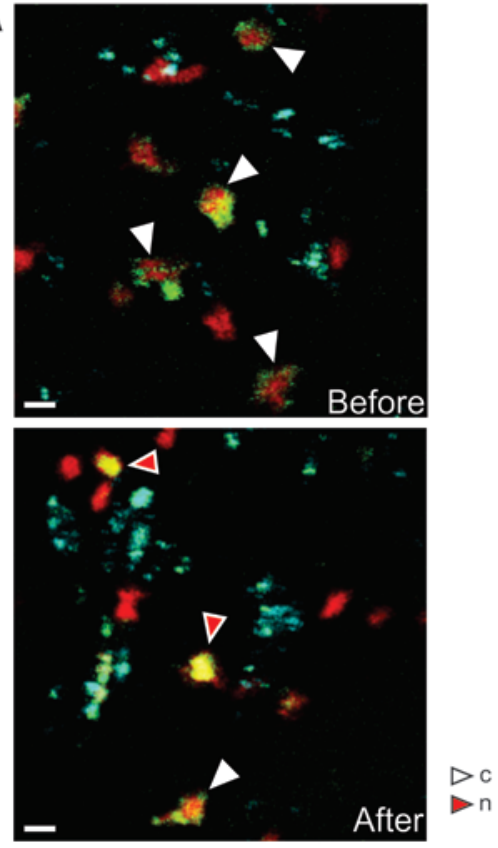

E

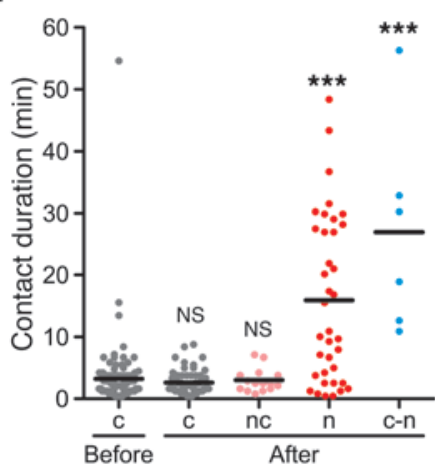

B
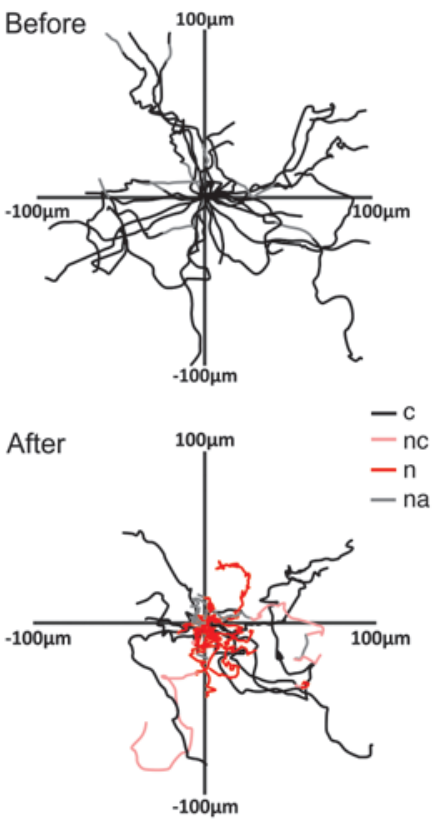

F

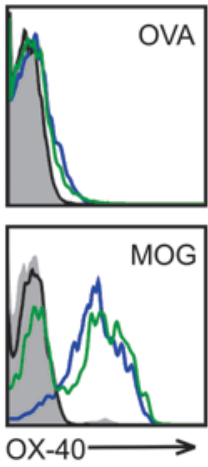

C

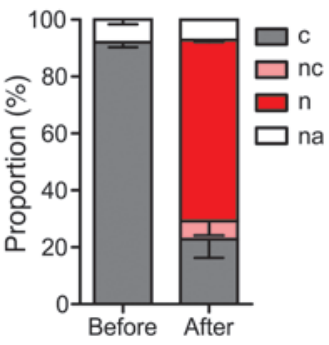

D

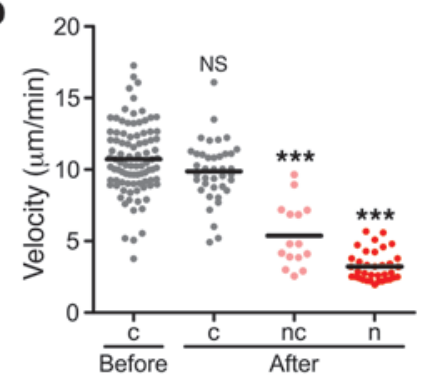

\section{Figure 6}

TMOG-NFAT-GFP cell activation by an exogenous antigen. (A) Representative still images of SNARF-labeled (red) TMOG-NFAT-GFP cells (green) interacting with local APCs (cyan) before and after local application of MOG into the imaging window. White and red arrowheads denote cytoplasmic and nuclear location, respectively. Scale bars: $10 \mu \mathrm{m}$. (B) Trajectory lines from representative videos before and after injection of soluble MOG (see Figure 4D). (C-E) $\triangle$ NFAT-GFP distribution pattern (C), velocity (D), and T cell/APC contact duration (E) in TMOG-NFAT-GFP cells analyzed before and after MOG application. Results from 3 independent experiments and at least 4 different videos per condition are shown. (F) Expression of the activation marker OX-40 on CNS-infiltrating TMOG-NFAT-GFP cells upon intrathecal application of OVA or MOG antigen, 3 days after their i.v. transfer. Expression was evaluated by flow cytometry on ex vivo isolated T cells. Representative results from 3 independent experiments are shown. ${ }^{* \star *} P<0.001,1$-way ANOVA followed by Kruskal-Wallis/Dunn multiple-comparison test.

shown in Figure 6F, the $\mathrm{T}_{\mathrm{MOG}}$ cells were activated only after soluble MOG treatment. Taken together, these results indicated that the increased availability of exogenous MOG changed the behavior of $\mathrm{T}_{\text {MOG-NFAT-GFP cells from an EAE }}{ }^{\text {lo }}$ to an $\mathrm{EAE}^{\text {hi }}$ pattern.

\section{Discussion}

Immune responses against foreign or self-antigens are the result of complex interactions between immune cells and their surrounding cellular milieu. These interactions centrally involve contacts between neighboring cells, and these contacts critically determine the character of the unfolding immune reaction. Approaches to characterize immune cell communication in different milieus include real-time imaging and isolation of the interacting partner cells for ex vivo analysis, but, owing to the lack of suitable indicators, direct visualization of contacts resulting in cell activation has been futile so far.

Our present study describes a genetic marker, $\triangle$ NFAT-GFP, that enables mapping - in vivo and in real time - the activation events in autoimmune $\mathrm{CD}^{+} \mathrm{T}$ cells passing through the $\mathrm{BBB}$ and the surrounding leptomeningeal space. $\triangle$ NFAT-GFP displayed qualities that appear to render it more suitable for 2-photon imaging than previously suggested activation indicators. Among these, TCR complex-related genetic markers, such as a CD3\}-GFP fusion protein (15-17), or linker for activation of T cells-GFP (LAT-GFP) fusion protein (18), showed $\mathrm{T}$ cell activation by clustering at the immunological synapses. However, due to relatively low vertical 
resolution of 2-photon microscopy (typically, 3- to 4- $\mu \mathrm{m}$ spacing), detection of small clusters within 3-dimensional volume is not optimal. In contrast, $\triangle$ NFAT-GFP subcellular localization was reliably detectable in more than $90 \%$ of the T cells during intravital 2-photon imaging. In transgenic mice, GFP expressed from the immediate early gene locus NUR77 was used as activation indicator (19). This system can distinguish the strength of TCR stimulation according to GFP expression level; however, the GFP protein needs to be transcribed and maturated, a process that takes at least few hours, too long for real-time imaging. $\triangle$ NFAT-GFP nuclear translocation, on the other hand, occurred within minutes upon stimulation. Finally, calcium indicators had been used to monitor $\mathrm{T}$ cell activation in vivo (20); however, small-molecule calcium indicators are rapidly pumped out from $\mathrm{T}$ cells and thus fail to label them over extended periods of time. Thus, $\triangle$ NFAT-GFP fusion protein qualifies as a reliable indicator to detect rapid $\mathrm{T}$ cell activation induced by antigen recognition in vivo.

The members of the NFAT family are expressed in a large number of tissues, most prominently in immune cells (21). In T cells, NFAT acts as a key factor in calcium-controlled gene transcription. After TCR engagement and intracellular calcium increase, calcineurin dephosphorylates NFAT, which then translocates from the cytosol to the nucleus, where - in cooperation with other transcription factors - it binds to DNA to initiate gene expression. However, although $\triangle$ NFAT-GFP is a reliable indicator of TCR/ calcium-dependent cell activation, it is not absolutely specific. Apart from TCR activation, the pathway may also be triggered via nonimmune receptors; conversely, $\mathrm{T}$ cells may be stimulated via alternative pathways, which would not be indicated by $\triangle$ NFATGFP (22). This must be considered in the case of the intraluminal $\mathrm{T}$ cell migration modes for which no $\triangle$ NFAT-GFP translocation was observed. The negative finding of NFAT transition may exclude classic TCR-mediated activation, but not necessarily distinct stimulatory pathways with or without TCR participation.

In the present study, we investigated several sets of myelin autoimmune T cells, sharing either genotype or antigen specificity, to correlate their behavior with their discrepant encephalitogenic potentials. Translocation of $\triangle$ NFAT-GFP was noted in the brain autoimmune $T$ cells that had crossed the cerebrovascular wall, but was strictly limited to $E E^{\text {hi }} \mathrm{T}$ cells. Nuclear $\triangle$ NFAT-GFP appeared in the EAE ${ }^{\text {hi }} \mathrm{T}_{\text {MBP-NFAT-GFP }}$ cells during and after contact with the local phagocytes. When isolated ex vivo, these phagocytes present locally produced myelin autoantigens to specific $\mathrm{T}$ cells, but the antigenic strength in vitro is weak. The responding $\mathrm{T}$ cells are activated to release cytokines, but do not proceed to mitosis unless supplemented with exogenous antigen (2). In contrast, the EAE ${ }^{\text {lo }}$ $\mathrm{T}_{\text {MOG-NFAT-GFP }}$ cells readily crossed the BBB, but failed to translocate $\triangle$ NFAT-GFP. The lack of reactivation coincided with a lack of long-term contacts with local antigen-presenting phagocytes. We suspect that this truncated response reflects decreased availability of MOG autoantigen in the Lewis rat, involving decreased local production (23) and/or suboptimal binding of MOG epitopes to relevant MHC class II molecules (24).

Imaging studies on the behavior of the $\mathrm{T}$ cells encountering exogenous antigens in secondary lymph organs have distinguished enduring stable contacts (synapses) from transient interactions (kinapses) (25). Formation of these contacts is determined by several factors, such as the available antigen dose (26), the nature of the APCs (macrophage-like APCs in the present study, instead of DCs, as reported previously; ref. 2), and the functional status of the T cells (27). According to this scheme, most of the APC contacts formed by EAE ${ }^{\text {hi }} \mathrm{T}$ cells in the leptomeningeal space qualify as kinapses (motility between 2.5 and $5.0 \mu \mathrm{m} / \mathrm{min}$ ), whereas synapses (motility slower than $2.5 \mu \mathrm{m} / \mathrm{min}$ ) were rarely observed. Both kinapses and synapses might be sufficient to translocate NFAT to the nucleus and to induce the transcription of activationdependent gene profiles (28). NFAT translocation and, implicitly, intracellular calcium increases are considered stop-signals to T cells (29). However, this was not cogently the case in the leptomeningeal $\mathrm{T}_{\text {MBP-NFAT-GFP }}$ cells, which continued visiting APCs successively in the activated, NFAT-translocated state. In fact, this behavior seemed to support our previous suggestion that contacts with leptomeningeal APCs serve to guide immigrant autoimmune $\mathrm{T}$ cells into the CNS parenchyma, rather than arresting them for extended periods of time.

$\triangle$ NFAT-GFP may pave the way to new strategies to directly study contact-dependent immune cell communication in response to foreign and self-antigens. These contacts are manifold and complex and may lead to cell activation or to silencing. As previously demonstrated by studies of EAE models, brain autoimmune T cells are activated in the gut (30), then pass through different milieus in lung and peripheral immune organs, where they are deactivated and reprogrammed (31), before finally passing through the cerebrovascular BBB (2). The use of $\triangle$ NFAT-GFP allows for distinguishing activating from other cell-to-cell events. Beyond its use for studying brain autoimmunity, this class of reagents may be applicable to any study of immune milieus, such as during development, infection, and tumor responses.

\section{Methods}

Animals. Lewis rats were obtained from and kept and bred in the animal facility at the Max Planck Institutes of Biochemistry/Neurobiology.

Establishment of GFP-labeled antigen-specific T cell lines. The antigen-specific $\mathrm{T}$ cell lines were established from the LNs of immunized animals, as described previously (32). Briefly, 6- to 8-week-old male rats were immunized with antigen/CFA. 10 days later, the LN cells were isolated and cultured together with GP+E-86 cells (ATCC no. CRL-9642), which were transfected to produce a GFP gene-coding retrovirus, pMSCVneo-GFP. Alternatively, GP+E-86 cells transfected with pMSCVneo- $\Delta$ NFAT-GFP (aa 1-460 of mouse NFAT1) were used. The T cells were positively selected by specific antigen stimulation and negatively using an antibiotic resistance gene included in the retroviral vector. At least 3 rounds of stimulation with the antigen and subsequent expansion in the conditional medium were completed before the cells were used for experiments. For EAE induction, $5 \times 10^{6}$ freshly stimulated $T$ cells were transferred into naive animals via the tail vein. Clinical scoring was as follows: 0 , no disease; 1 , flaccid tail; 2 , gait disturbance; 3, complete hind limb paralysis; 4, tetraparesis.

Flow cytometric analysis. T cells were purified th the peak of infiltration from CNS meninges or parenchyma as described previously (2). Briefly, meninges and parenchyma were carefully dissected, and single-cell suspensions were prepared by passing through a $70-\mu \mathrm{m}$ cell strainer. To purify lymphocytes from the CNS parenchyma, Percoll (GE Healthcare) gradient was used. No further purification was necessary to recover $T$ cells from meninges. The cells were stained with activation marker, CD134 (OX-40; Serotec), followed by rabbit anti-mouse IgG-PerCP (Santa Cruz Biotechnology). Staining was evaluated by FACSCalibur operated by Cell Quest software (Becton Dickinson). The obtained results were processed by Flow Jo 7.6 software (TreeStar).

Confocal microscopy. In vitro cultured resting $\mathrm{T}_{\text {MBP-NFAT-GFP }}$ cells were labeled with PKH26 Red Fluorescent Cell linker kit for general cell mem- 
brane labeling (Sigma-Aldrich). Some cells were treated with $1 \mu \mathrm{M}$ ionomycin for 3 minutes, after which stimulated and nonstimulated cells were incubated on collagen-coated glass slides for 15 minutes to immobilize them. Cells were fixed in $4 \%$ PFA and stained with $5 \mu \mathrm{g} / \mathrm{ml}$ DAPI for 10 minutes. Images were taken with $\times 40$ oil-immersion objective (NA 1.25) using Leica SP2 confocal microscopy.

Fluorescent videomicroscopy. Resting in vitro $\mathrm{T}_{\mathrm{MBP} \text {-NFAT-GFP }}$ cells were incubated in a $\mu$-slide $\mathrm{I}^{0.4}$ collagen-coated Microscopy Chamber (ibidi) for imaging. T cells were stimulated with $1 \mu \mathrm{M}$ ionomycin during image acquisition, and imaging continued for 15 minutes after stimulation. Cells were then collected, washed of ionomycin, plated again in a new Microscopy Chamber, and imaged for another 2-3 hours. Time-lapse recordings were performed using an inverted microscope (Axiovert 200M) equipped with $\times 40$ oil-immersion objective (NA 1.3; Zeiss). Images were acquired using a Coolsnap-HQ camera (Photometrics; Roper Scientific) and processed by MetaMorph (Visitron Systems).

SNARF-1 labeling. $\triangle$ NFAT-GFP-labeled "migratory" T cells were prepared from host spleens 3 days after transfer. The erythrocytes were removed from the suspensions by osmotic lysis, and the macrophages by adhesion on culture dishes. Prior to SNARF-1 labeling, we enriched for $\mathrm{CD}^{+} \mathrm{T}$ cells using the MagCellect* Rat $\mathrm{CD} 4^{+} \mathrm{T}$ Cell Isolation Kit (R\&D Systems). On average, $20 \%-30 \%$ of the T cells were $\triangle$ NFAT-GFP-expressing cells. The T cells were labeled with SNARF-1 (Invitrogen) by incubating for 15 minutes at a concentration of $1.25 \mu \mathrm{M}$. SNARF-1 labeling results in a strong signal in the nucleus, whereas cytosol staining is often weaker; therefore, $\triangle$ NFAT-GFP in the cytosol may be detected without SNARF-1 counterstaining.

Intravital imaging. The surgical procedures and technical setup of the 2 -photon microscopy were largely as described previously $(2,33)$. For excitation, the pulsed laser was tuned to 880 or $935 \mathrm{~nm}$, and imaging was performed using a $\times 20$ water-immersion objective (NA 0.95 ; Olympus) or a $\times 25$ water-immersion objective (NA 0.95; Leica). The emitted photons were collected using nondescanned photomultiplier tubes. Typically, imaging was performed at a 40- to $50-\mu \mathrm{m}$ stack height (3- to $4-\mu \mathrm{m} z$ step) in approximately 25 -second intervals.

Image analysis. The position of the $\mathrm{T}$ cells in the 3-dimensional space was analyzed using Imaris software. Calculation of mean square displacement was described previously (34). T cell/APC contacts were analyzed manually; all physical interactions between T cells and labeled meningeal APCs in the 3-dimensional volume were defined as contacts. All representative videos were processed using ImageJ software. In some videos, the contrast was adjusted by liner rescaling, and the noise was removed using a Median filter and Gaussian blur.

Injection of exogenous antigen. Soluble MOG was injected 3 days after i.v. transfer of $\mathrm{T}_{\text {MOG-NFAT-GFP }}$ cells. Intrathecal injection into the cisterna magna was performed as described previously (2). Alternatively, during intravital imaging, MOG was instilled locally via a microcapillary (tip diameter, $13 \mu \mathrm{m}$; Biomedical Instruments) that was positioned using a microcapillary manipulator (Luigs \& Neumann). The capillary was pushed through the arachnoidea through a hole cut by a high-energy 2-photon laser. For the injection, the microcapillary was filled with a solution of $10 \mu \mathrm{g} / \mathrm{ml}$ MOG protein and $0.2 \mu \mathrm{g} / \mathrm{ml}$ tetramethylrhodamine dextran. We injected a total volume of $3-4 \mu \mathrm{l}$ of the solution.

Statistics. Statistical evaluation was performed using Prism software (GraphPad). In all experiments, Mann-Whitney nonparametric $t$ test (2-tailed) was used for 1 pair, and 1-way ANOVA followed by Kruskal-Wallis test/Dunn's multiple-comparison test was used for more than 1 pair. A $P$ value less than 0.05 was considered significant.

Study approval. All experiments were conducted according to the Bavarian state regulations for animal experimentation and were approved by the appropriate authorities.

\section{Acknowledgments}

We thank Sabine Kosin and Marko Lazic for technical support. This work was supported by the Max Planck Society, LudwigMaximilians University München, Deutsche Forschungsgemeinschaft (SFB571, projects C6 and B10), Hertie Foundation, Novartis Foundation for Therapeutic Research, and German Academic Exchange service (DAAD).

Received for publication October 5, 2012, and accepted in revised form December 6, 2012.

Address correspondence to: Naoto Kawakami or Hartmut Wekerle, Max-Lebsche-Platz 31, 81377 Munich, Germany. Phone: 49.89.7095.8386; Fax: 49.89.7095.8380; E-mail: Naoto.Kawakami@ med.uni-muenchen.de (N. Kawakami), hwekerle@neuro.mpg.de (H. Wekerle).
1. Sorokin L. The impact of the extracellular matrix on inflammation. Nat Rev Immunol. 2010; 10(10):712-723.

2. Bartholomäus I, et al. Effector T cell interactions with meningeal vascular structures in nascent autoimmune CNS lesions. Nature. 2009;462(7269):94-98.

3. Kivisäkk P, et al. Localizing central nervous system immune surveillance: Meningeal antigen-presenting cells activate $\mathrm{T}$ cells during experimental autoimmune encephalomyelitis. Ann Neurol. 2009; 65(4):457-469.

4. Odoardi F, Kawakami N, Klinkert WEF, Wekerle $\mathrm{H}$, Flügel A. Blood-borne soluble protein antigen intensifies $\mathrm{T}$ cell activation in autoimmune CNS lesions and exacerbates clinical disease. Proc Natl Acad Sci U S A. 2007;104(47):18625-18630.

5 . Kawakami N, et al. The activation status of neuroantigen-specific $\mathrm{T}$ cells in the target organ determines the clinical outcome of autoimmune encephalomyelitis. J Exp Med. 2004;199(2):185-197.

6. Flügel A, Bradl M. New tools to trace populations of inflammatory cells in the CNS. Glia. 2001. 36(2):125-136

7. Hogan PG, Chen L, Nardone J, Rao A. Transcriptional regulation by calcium, calcineurin, and NFAT. Genes Dev. 2003;17(18):2205-2232.
8. Okamura H, et al. Concerted dephosphorylation of the transcription factor NFAT1 induces a conformational switch that regulates transcriptional activity. Mol Cell. 2000;6(3):539-550.

9. Aramburu J, Garcia-Cozar F, Raghavan A, Okamura H, Rao A, Hogan PG. Selective inhibition of NFAT activation by a peptide spanning the calcineurin targeting site of NFAT. Mol Cell. 1998;1(5):627-637.

10. Shaw KT, et al. Immunosuppressive drugs prevent a rapid dephosphorylation of transcription factor NFAT1 in stimulated immune cells. Proc Natl Acad SciU S A. 1995;92(24):11205-11209.

11. Piccio L, et al. Molecular mechanisms involved in lymphocyte recruitment in inflamed brain microvessels: Critical roles for P-selectin glycoprotein ligand-1 and heterotrimeric G(i)-linked receptors. Jimmunol. 2002;168(4):1940-1949.

12. Engelhardt B. Molecular mechanisms involved in $\mathrm{T}$ cell migration across the blood-brain barrier. J Neural Transm. 2006;113(4):477-485.

13. Bousso P, Bhakta NR, Lewis RS, Robey E. Dynamics of thymocyte-stromal cell interactions visualized by two-photon microscopy. Science. 2002; 296(5574):1876-1880.

14. Flügel A, et al. Migratory activity and functional changes of green fluorescent effector $T$ cells before and during experimental autoimmune encephalomyelitis. Immunity. 2001;14(5):547-560.

15. Yudushkin IA, Vale RD. Imaging T-cell receptor activation reveals accumulation of tyrosine-phosphorylated CD3zeta in the endosomal compartment. Proc Natl Acad Sci U S A. 2010;107(51):22128-22133.

16. Huppa JB, Gleimer M, Sumen C, Davis MM. Continuous $T$ cell receptor signaling required for synapse maintenance and full effector potential. Nat Immunol. 2003;4(8):749-755.

17. Richie LI, Ebert PJR, Wu LC, Krummel MF, Owen JJT, Davis MM. Imaging synapse formation during thymocyte selection: Inability of CD3 to form a stable central accumulation during negative selection. Immunity. 2002;16(4):595-606.

18. Tanimura N, et al. Dynamic changes in the mobility of LAT in aggregated lipid rafts upon T cell activation. J Cell Biol. 2003;160(1):125-135.

19. Moran AE, et al. T cell receptor signal strength in Treg and iNKT cell development demonstrated by a novel fluorescent reporter mouse. J Exp Med. 2011;208(6):1279-1289.

20. Wei SH, Safrina O, Yu Y, Garrod KR, Cahalan MD, Parker I. Ca2+ signals in CD4+ T cells during early contacts with antigen-bearing dendritic cells in lymph node. J Immunol. 2007;179(3):1586-1594. 
21. Crabtree GR, Olson EN. NFAT signaling: Choreographing the social lives of cells. Cell. 2002; 109(suppl):S67-S79.

22. Macian F. NFAT proteins: Key regulators of T-cell development and function. Nat Rev Immunol. 2005; 5(6):472-484.

23. Pagany M, Jagodic M, Bourquin C, Olsson T, Linington $\mathrm{C}$. Genetic variation in myelin oligodendrocyte glycoprotein expression and susceptibility to experimental autoimmune encephalomyelitis. J Neuroimmunol. 2003;139(1-2):1-8.

24. Weissert R, et al. MHC haplotype dependent regulation of MOG induced EAE in rats. J Clin Invest. 1998; 102(6):1265-1273.

25. Dustin ML. T-cell activation through immunological synapses and kinapses. Immunol Rev. 2008; 221:77-89.
26. Skokos D, et al. Peptide-MHC potency governs dynamic interactions between $\mathrm{T}$ cells and dendritic cells in lymph nodes. Nat Immunol. 2007; 8(8):835-844.

27. Garcia Z, Pradelli E, Celli S, Beuneu H, Simon A, Bousso P. Competition for antigen determines the stability of T cell-dendritic cell interactions during clonal expansion. Proc Natl Acad Sci U S A. 2007; 104(11):4553-4558

28. Moreau HD, et al. Dynamic in situ cytometry uncovers $\mathrm{T}$ cell receptor signaling during immunological synapses and kinapses in vivo. Immunity. 2012;37(2):351-363.

29. Negulescu PA, Krasieva TB, Khan A, Kerschbaum $\mathrm{HH}$, Cahalan MD. Polarity of T cell shape, motility, and sensitivity to antigen. Immunity. 1996; 4(5):421-430.
30. Berer K, et al. Commensal microbiota and myelin autoantigen cooperate to trigger autoimmune demyelination. Nature. 2011;479(7374):538-541.

31. Odoardi $\mathrm{F}$, et al. $\mathrm{T}$ cells become licensed in the lung to enter the central nervous system. Nature. 2012;488(7413):675-679.

32. Flügel A, Willem M, Berkowicz T, Wekerle H. Gene transfer into CD4 + T lymphocytes: Green fluorescent protein engineered, encephalitogenic $T$ cells used to illuminate immune responses in the brain. Nat Med. 1999;5(7):843-847.

33. Odoardi F, et al. Instant effect of soluble antigen on effector $\mathrm{T}$ cells in peripheral immune organs during immunotherapy of autoimmune encephalomyelitis. Proc Natl Acad Sci U S A. 2007;104(3):920-925.

34. Beltman JB, Marée AF, de Boer RJ. Analysing immune cell migration. Nat Rev Immunol. 2009;9(11):789-798. 